\title{
An Adaptive Howling Canceller Using 2-Tap Linear Predictor
}

\author{
Akira Sogami, Yosuke Sugiura, Arata Kawamura*, Youji Iiguni \\ Department of Systems Innovation, Graduate School of Engineering Science, Osaka University, Toyonaka, Japan \\ Email: *kawamura@sys.es.osaka-u.ac.jp
}

Received October 1, 2012; revised November 1, 2012; accepted November 8, 2012

\begin{abstract}
This paper proposes an adaptive howling canceller using notch filter and 2-tap linear predictor, where howling consists of a single sinusoidal signal whose magnitude is much greater than other frequency's magnitudes. The employed 2-tap linear predictor can quickly detect howling due to its high convergence speed. Although the output signal of the 2-tap linear predictor cannot be directly used as one of a howling canceller, we can obtain the frequency of howling from the filter coefficient. We utilize the filter coefficient of the 2-tap linear predictor to design a notch filter which achieves a very narrow elimination band. The designed notch filter removes only howling and retains other desired signals. Simulation results show that the proposed adaptive howling canceller can quickly detect and effectively remove howling.
\end{abstract}

Keywords: Howling Canceller; Linear Predictor; Notch Filter; Single Sinusoidal Signal; Convergence Speed

\section{Introduction}

Howling is an annoying and persistence problem which is mainly caused in public address system. The public address system amplifies a signal observed at a microphone and transmits the amplified signal with a loudspeaker. Then, there exists a feedback path from the loudspeaker to the microphone. This feedback path forms an acoustical closed loop. When a frequency amplitude response of the closed loop is greater than 1 and its phase response is $2 \pi$, howling is caused, i.e., a single sinusoidal signal rapidly becomes large. In this case, audience cannot avoid perception of this unpleasant sound, and also it is difficult to receive a desired signal. Moreover, the public address system often breaks down due to howling. To avoid this undesired phenomenon, many approaches have been studied. One of the most famous and effective methods is an echo canceller [1]. The echo canceller adaptively estimates the acoustical impulse response from the loudspeaker to the microphone, and provides a replica of the feedback signal. When the echo canceller perfectly estimates the feedback impulse response, subtracting the replica of the feedback signal from the observed signal gives perfect suppression of the feedback signal and thus howling is not caused. Since the acoustical impulse response is time-variant, the echo canceller is required to achieve both of high estimation accuracy and high convergence speed. Unfortunately, a tread-off exists between the convergence speed and the estimation accu-

${ }^{*}$ Corresponding author. racy. Actually, in many practical environments, the echo canceller cannot work well and hence it often causes degradation of sound quality, echo, or howling. Sogami et al. have proposed a simple howling canceller [2-3] which estimates howling by utilizing only the distance information between the loudspeaker and the microphone. This method can estimate the frequency of howling faster than the conventional echo canceller, under the assumption that howling is depending only on the direct distance from the loudspeaker to the microphone. Although howling often depends on the direct distance, the above assumption cannot hold for howling which depends on the distance including reflections. Actually, all the distances including reflections cannot be calculated. These conventional researches imply that the occurrence of howling may not be avoided and thus we have to prepare another howling canceller which adaptively removes howling as fast as possible after it is caused.

To remove howling after its occurrence, adaptive notch filter techniques are useful [4-10]. They achieve very narrow elimination bandwidth, and automatically estimate and remove a single sinusoidal signal like howling. Unfortunately, it also has a trade-off problem between the convergence speed and the estimation accuracy. Efficient notch filters which can solve the trade-off problem have been proposed [11-13]. Their main idea is to employ two or more notch filters, where one notch filter achieves high estimation accuracy at an expense of convergence speed, and the other notch filter has high convergence speed with low estimation accuracy. In such 
techniques, addition to an increase of their computational cost, it may not achieve an accurate adaptation because the notch filter's impulse responses are long basically.

In this paper, we propose an adaptive howling canceller which achieves both of high convergence speed and high estimation accuracy to remove howling. The proposed method consists of a notch filter and a 2-tap linear predictor. Although the proposed method utilizes the additional filter, the 2-tap linear predictor updates only one filter coefficient to detect howling. With its short impulse response, the 2-tap linear predictor can quickly evaluate the prediction error signal in the steady-state for adaptation. Since the filter coefficient has the information of the frequency of howling after convergence, we easily design the notch filter whose elimination frequency is identical to the frequency of howling. Then, we can remove howling quickly and effectively. The additional computational cost of the proposed method is minimal among conventional adaptive notch filters which employ two or more additional notch filters. Simulation results show that the proposed howling canceler effectively removes howling.

\section{Howling Canceller with Notch Filter}

Let consider a public address system shown in Figure 1, where $s(n)$ denotes the source signal produced by human in general, and $y(n)$ is the feedback signal from the loudspeaker to the microphone. The observed signal is represented as

$$
x(n)=s(n)+y(n) .
$$

The observed signal is amplified by the attenuator so that $\tilde{s}(n)=a x(n)$, where $a$ is a constant. The amplified signal $\tilde{s}(n)$ is produced from the loudspeaker. The signal $\tilde{s}(n)$ is received at the microphone after passing through the unknown acoustical feedback path whose transfer function is $P(z)$. Then, the closed loop is formed as shown in Figure 1. When the amplitude frequency response of the closed loop is greater than 1 and the phase frequency response is $2 \pi$ for a certain frequency, howling will occur at the corresponding frequency. Figure 2 shows an example of the occurrence of howling. Here, we set the acoustical impulse response as

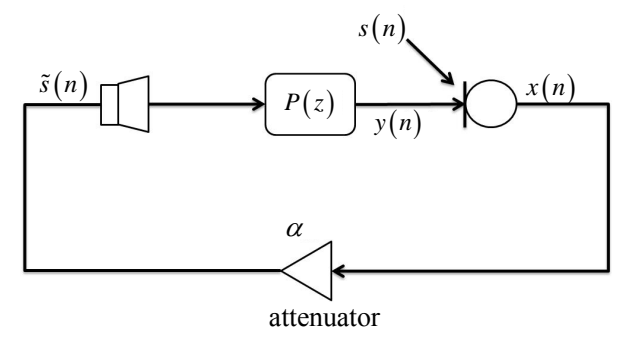

Figure 1. Public address system. uniform random variables. The top panel shows the waveform of $s(n)$, and the middle panel shows the transmitted signal $\tilde{s}(n)$. In this simulation, we gradually increased $a$. As a result, howling occurred at around 30,000 samples. An expanded waveform is shown in the bottom panel. We see from this result that howling explosively increases and we should remove it as fast as possible.

First, we explain the standard adaptive notch filter to remove howling. The notch filter passes all frequencies expect of the narrow frequency band whose center frequency is called as the notch frequency. The elimination bandwidth and the notch frequency can be individually designed [4-10]. The transfer function of the notch filter $N(z)$ is given by [6-8]

$$
N(z)=\frac{1}{2}\left(1+\frac{r+\alpha z^{-1}+z^{-2}}{1+\alpha z^{-1}+r z^{-2}}\right),
$$

where $\alpha$ is a parameter to design the notch frequency and $r(-1<r<1)$ determines the elimination bandwidth. The relation between $\alpha$ and the notch frequency is given as

$$
\alpha=-(1+r) \cos \left(2 \pi \frac{F}{F_{S}}\right),
$$

where $F[\mathrm{~Hz}]$ denotes the notch frequency and $F_{S}[\mathrm{~Hz}]$ denotes the sampling frequency. The relation of $r$ and the elimination bandwidth $K[\mathrm{~Hz}]$ is represented as

$$
r=\frac{1+\cos \left(2 \pi K / F_{S}\right)-\sin \left(2 \pi K / F_{S}\right)}{1+\cos \left(2 \pi K / F_{S}\right)+\sin \left(2 \pi K / F_{S}\right)} .
$$

Figure 3 shows the structure of the notch filter, where $x(n)$ is the input signal, and $\tilde{x}(n)$ is the output signal. The notch filter includes the IIR unit, and hence its impulse response is infinite. Figure 4 shows the frequency amplitude response of the notch filter $N(z)$ when $\alpha=0\left(F=F_{S} / 2\right)$ with $r=0.8,0.9,0.99$, where the vertical axis denotes the amplitude response and the

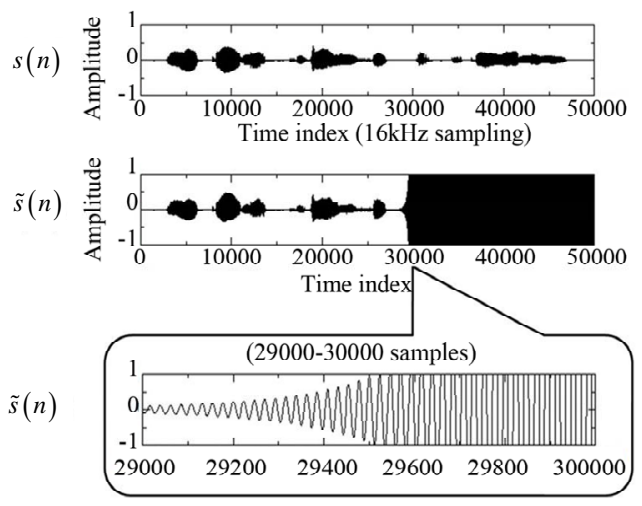

Figure 2. Example of howling. 


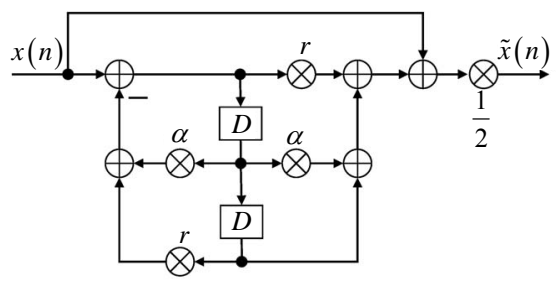

Figure 3. Structure of notch filter.

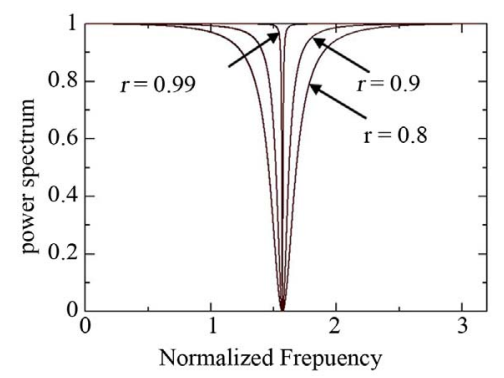

Figure 4. Frequency amplitude response of notch filter.

horizontal axis denotes the normalized frequency. We see from Figure 4 that the elimination bandwidth becomes narrow with increasing $r$ toward to 1 . Thus, we can remove only howling when setting $r$ close to 1 .

The frequency of howling is usually unknown. Hence, we have to adaptively estimate and remove it. When the observed signal includes a sinusoidal signal whose magnitude is much greater than the other frequency's magnitudes, an adaptive notch filter can automatically estimate and remove the single sinusoidal signal when its coefficient $\alpha$ is updated by a gradient method [4]. The coefficient $\alpha$ converges so that the notch filter's output power is minimized. Since howling can be approximated such as the sinusoidal signal, the adaptive notch filter can automatically detect and remove howling.

However, the gradient method includes an annoying trade-off problem between convergence speed and estimation accuracy. To accelerate convergence speed with remaining high estimation accuracy, in many approaches, an additional adaptive notch filter is introduced [11-13], where the main notch filter has high estimation accuracy and the other notch filter has high convergence speed. Comparing their output signals, we can choose better one. This method is useful when the notch filter is appropriately updated by using the output signal in the steady state. Unfortunately, such update is difficult, because notch filter's impulse responses are generally long.

\section{Howling Canceller with Notch Filter and 2-Tap Linear Predictor}

In this section, we propose an adaptive howling canceller which utilizes an adaptive notch filter and an additional 2-tap linear predictor, where the proposed method achieves both of high convergence speed and high estimation accuracy.

As mentioned above sections, howling can be approximated as a single sinusoidal signal whose magnitude is much greater than other frequency's magnitudes. Our purpose is to detect the frequency of howling as fast as possible. A linear predictor, often called as an adaptive line enhancer [1], is useful to extract a sinusoidal signal from the sinusoidal signal embedded in a wideband signal. Hence, the estimation error signal of the linear predictor does not include the sinusoid. Intuitively, we note that the estimation error signal can be utilized as the output signal of a howling canceller. Unfortunately, to accurately remove howling only, the filter length of the linear predictor must be long. In actual, the notch filter is the most effective filter to remove howling, because of its low computational complexity and steep frequency response. In the use of the adaptive notch filter, we encounter the trade-off problem again.

To solve this problem, we propose a combination method of a linear predictor and a notch filter. The employed linear predictor has the minimum impulse response which is just 2 samples (called 2-tap linear predictor) to detect the frequency of howling. The notch filter is adaptively designed from the 2-tap linear predictor's coefficient to align the notch frequency with the frequency of howling. Since the 2-tap linear predictor can achieve very short impulse response, we can quickly obtain its prediction error signal in the steady state for adaptation. It means that the 2-tap linear predictor achieves high convergence speed and we obtain the frequency of howling quickly.

Figure 5 shows the 2-tap linear predictor, where $\hat{x}(n)$ and $e(n)$ denote the predicting signal and the prediction error signal, respectively. The 2-tap linear predictor provides a replica of the present input signal by linear combination of past two input samples with two coefficients as $\hat{x}(n)=h_{1}(n) \times(n-1)+h_{2}(n) \times(n-2)$. Here, $h_{1}(n)$ and $h_{2}(n)$ denote the 1 st and 2 nd filter coefficients, respectively, and the replica has inverse phase of the present input signal. We update the 2-tap linear predictor so that the power of the following prediction error signal is minimized.

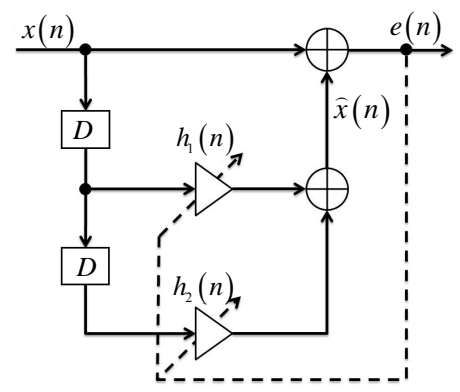

Figure 5. 2-tap linear predictor. 


$$
e(n)=x(n)+\hat{x}(n)=1+h_{1}(n) x(n-1)+h_{2}(n) x(n-2)
$$

Under the assumption that howling can be expressed as $x(n) \approx p_{1} \cos \left(2 \pi F_{1} / F_{S} n+\theta_{1}\right)$, where $F_{1}, p_{1}, \theta_{1}$ are the frequency of howling, amplitude, and phase, respectively, the 2-tap linear predictor can estimate the single sinusoidal signal by using a gradient method [3-10]. It is well known that the 2-tap linear predictor for such single sinusoidal input signal converges so that

$$
h_{1}(n) \rightarrow-2 \cos \left(2 \pi \frac{F_{1}}{F_{S}}\right), h_{2}(n) \rightarrow 1 .
$$

Here, we note that the 1 st coefficient $h_{1}(n)$ depends on the frequency of howling, while the 2 nd coefficient $h_{2}(n)$ is independent with the input signal. It implies that we do not need to update the 2nd coefficient when predicting howling. Specifically, we can fix the 2 nd coefficient as $h_{2}(n)=1$. On the other hand, we have to update $h_{1}(n)$ to obtain the frequency of howling. After convergence, $h_{1}(n)$ is used to design the notch filter to remove howling. When the notch frequency is accurately designed, the notch filter successfully cancels howling. Comparing (3) and (6), we have the following simple relation.

$$
\alpha=\frac{1+r}{2} h_{1}(n)
$$

The accurate $h_{1}(n)$ gives the accurate $\alpha$. Then, the notch filter with (7) completely cancels howling.

Figure 6 shows the proposed howling canceller, where LP denotes the 2-tap linear predictor. In the proposed system, $h_{1}(n)$ is updated by using the NLMS type algorithm given as

$$
h_{1}(n+1)=h_{1}(n)-\mu \frac{e(n) x(n-1)}{E\left[x^{2}(n-1)\right]},
$$

where $\mu$ is the step-size for adaptation. The notch filter's coefficient $\alpha$ is updated with (7), where $r$ and $h_{2}(n)$ are fixed.

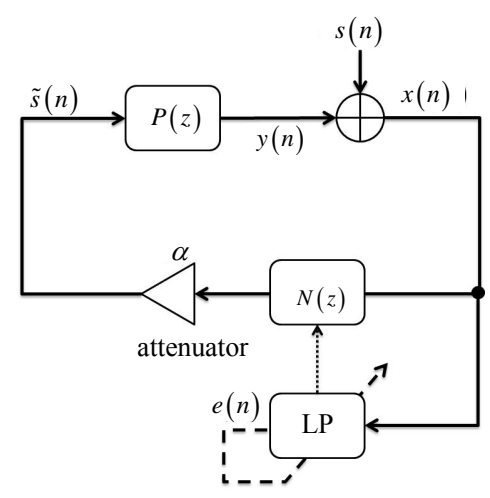

Figure 6. Proposed system.

\section{Simulation}

We carried out computer simulation to confirm the effectiveness of the proposed method. Here, we set feedback pass $P(z)$ as an FIR filter whose impulse response is set as a uniform random signal, where the order of the FIR filter is 50 . We also set the attenuator as $\alpha=4$ to satisfy the condition for occurrence of howling, and the step-size used in (8) as $\mu=0.05$. The source signal was a female voice signal sampled at $16 \mathrm{kHz}$. Since the expectation value $E\left[x^{2}(n-1)\right]$ shown in (8) cannot be calculated, we used a time averaged value defined as

$$
\overline{x^{2}(n-1)}=\frac{1}{M} \sum_{m=0}^{M-1} x^{2}(n-m),
$$

instead of $E\left[x^{2}(n-1)\right]$, where we set $M=50$. To compare the howling cancelation capability of the proposed method, we also performed the computer simulation for the conventional adaptive notch filter [4].

Figure 7 shows the results of howling cancellation, where Figure $7(\mathbf{a})$ shows the source signal $s(n)$, Figure 7(b) shows the output signal $\tilde{s}(n)$ without a howling canceller, Figure 7(c) denotes the output signal of the conventional adaptive notch filter, and Figure 7(d) shows the output signal of the proposed method. We see from Figure 7(b) that the waveform is explosively increasing when the howling canceller did not exist. Figure 7(c) shows that the conventional adaptive notch filter can remove howling. But, its convergence speed is comparatively slow, and thus we did not avoid the perception of howling. On the other hand, Figure 7(d) shows that the proposed method can effectively cancel howling. The difference between Figures 7(c) and (d) expresses the difference of respective convergence speeds. The proposed method removed howling faster than the conventional notch filter.

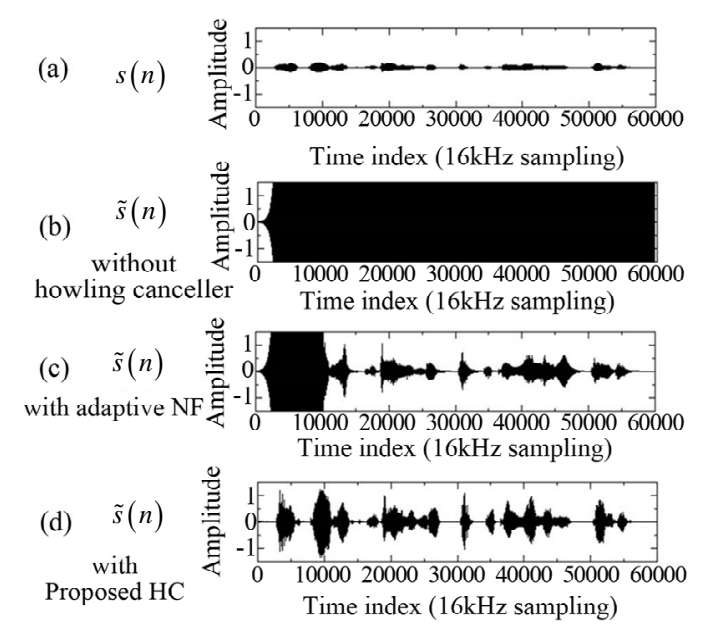

Figure 7. Waveforms of simulation result. (a) Source signal; (b) Loudspeaker output without howling canceller; (c) Loudspeaker output with conventional notch filter; (d) Loudspeaker output with proposed howling canceller. 


\section{Conclusion}

This paper has proposed a howling canceller which employs a 2-tap linear predictor and a notch filter. The 2-tap linear predictor is for estimating the frequency of howling, and the notch filter is for eliminating howling. Since the coefficient of the 2-tap linear predictor is simply transformed to the coefficient of the notch filter, we can easily update the notch filter according to the linear predictor. The simulation result showed the effectiveness of the proposed method.

\section{REFERENCES}

[1] S. Haykin, "Introduction to Adaptive Filters," Macmillan Publishing Company, New York, 1984.

[2] A. Sogami, A. Kawamura and Y. Iiguni, "Improvement of Speech Quality in Distance-Based Howling Canceller," IEICE Transactions on Fundamentals, Vol. 92, No. 4, 2009, pp. 1039-1046.

[3] A. Soagami, A. Kawamura and Y. Iiguni, "A High Speech Quality Distance-Based Howling Canceller with Adaptive Cascade Notch Filter and Silent Pilot Signal," IEICE Transactions on Fundamentals, Vol. 94, No. 11, 2011, pp. 2306-2314.

[4] S. Nishimura, "An Improved Adaptive Notch Filter for Detection of Multiple Sinusoids," IEICE Transactions on Fundamentals, Vol. 77, No. 6, 1994, pp. 950-955.

[5] A. Nehorai, "A Minimal Parameter Adaptive Notch Filter with Constrained Poles and Zeros," IEEE Transactions on Acoustics, Speech and Signal Processing, Vol. 33, No. 4, 1985, pp. 983-996. doi:10.1109/TASSP.1985.1164643

[6] H. C. Chong and U. L. Sang, "Adaptive Line Enhancement by Using an IIR Lattice Notch Filter," IEEE Trans- actions on Acoustics, Speech and Signal Processing, Vol. 37, No. 4, 1989, pp. 585-589. doi:10.1109/29.17543

[7] C. C. Tseng and S. C. Pei, "IIR Multiple Notch Filter Design Based on Allpass Filter," IEEE Transactions on Circuits and Systems II: Analog and Digital Signal Processing, Vol. 44, No. 2, 1997, pp. 133-136.

[8] S. C. Pei, W. S. Lu and C. C. Tseng, "Analytical TwoDimensional IIR Notch Filter Design Using Outer Product Expansion," IEEE Transactions on Circuits and Systems II: Analog and Digital Signal Processing, Vol. 44, No. 9, 1997, pp. 765-768.

[9] Y. V. Joshi and S. C. D. Roy, "Design of IIR Multiple Notch Filters Based on All-Pass Filters," IEEE Transactions on Circuits and Systems II: Analog and Digital Signal Processing, Vol. 46, No. 2, 1999, pp. 134-138.

[10] V. DeBrunner, "An Adaptive, High-Order, Notch Filter Using All Pass Sections," IEEE International Conference on Acoustics, Speech and Signal Processing, Vol. 3, 1998, pp. 1477-1480.

[11] Y. C. Lim, Y. X. Zou and N. Zheng, "A Piloted Adaptive Notch Filter," IEEE Transactions on Signal Processing, Vol. 53, No. 4, 2005, pp. 1310-1323. doi:10.1109/TSP.2005.843742

[12] A. Kawamura, Y. Itoh, J. Okello, M. Kobayashi and Y. Fukui, "Parallel Composition Based Adaptive Notch Filter: Performance and Analysis," IEICE Transactions on Fundamentals, Vol. 87, No. 7, 2004, pp. 1747-1755.

[13] A. Kawamura, Y. Iiguni and Y. Itoh, "An Adaptive Algorithm with Variable Step-Size for Parallel Notch Filter," IEICE Transactions on Fundamentals, Vol. 89, No. 2, 2006, pp. 511-519. 